E3S Web of Conferences 1, 37001 (2013)

DOI: $10.1051 / \mathrm{e} 3$ sconf/20130137001

(C) Owned by the authors, published by EDP Sciences, 2013

\title{
Determination of Trace Amounts of Lead with ETAAS After Single Drop Microextraction and Dispersive Liquid Liquid Microextraction Methods
}

\author{
M. Efeçınar ${ }^{1}$, P. Çakır ${ }^{2}$ and N. Şatıroğlu ${ }^{3}$ \\ ${ }^{1}$ Hacettepe University, Chemistry Department, 06800 Beytepe, Ankara, Turkey, melise@ hacettepe.edu.tr \\ ${ }^{2}$ Hacettepe University, Chemistry Department, 06800 Beytepe, Ankara, Turkey, pinarcakir.pc@ gmail.com \\ ${ }^{3}$ Hacettepe University, Chemistry Department, 06800 Beytepe, Ankara, Turkey, nurayo@ hacettepe.edu.tr
}

\begin{abstract}
Two liquid-phase microextraction procedures, single-drop microextraction (SDME) and dispersive liquid-liquid microextraction (DLLME), have been developed for the determination of lead by electrothermal atomic absorption spectrometry (ETAAS). Both methods were based on the formation of lead iodideRhodamine B complex which is in phosphoric acid medium. In the presence of KI, anionic lead iodide was complexed with Rhodamine B as an ion-association complex. Several factors that may be affected on the SDME and DLLME methods were optimized. In the optimum experimental conditions, the limit of detection (3s) and the enhancement factor were $0.008 \mu \mathrm{gL}^{-1}$ and 152 for SDME and $0.0129 \mu \mathrm{gL}^{-1}$ and 89 for DLLME respectively. The relative standard deviation (RSD) for eight replicate determinations of $0.25 \mu \mathrm{gL}^{-1} \mathrm{~Pb}$ was 4.6\% for SDME and $0.5 \mu \mathrm{gL}^{-1} \mathrm{~Pb}$ was $2.9 \%$ for DLLME. The developed methods were validated by the analysis of certified reference materials, and applied successfully to the determination of lead in several water and food samples.
\end{abstract}

Keywords: Lead, single drop microextraction, dispersive liquid liquid microextraction, ETAAS

\section{Introduction}

Heavy metals are very toxic, as ions or in compounds. They are soluble in water and may be readily absorbed into living organisms. The interest in the determination of trace heavy metal ions, such as lead in natural waters has increased because of the environmental problems and public health studies (Ming-Ho, 2005). The determination of trace metal ions in water samples requires the use of highly sensitive analytical techniques. Only few techniques have the sensitivity required to directly determine low quantities of analyte, such as electrothermal atomic absorption spectrometry (ETAAS) (Wang et al., 2007), inductively coupled plasma atomic emission spectrometry (ICP-AES) (Aceto et al., 2002) and inductively coupled plasma mass spectrometry (ICPMS) (Xia et al., 2008). AAS has been the most popular analytical technique for the determination of metallic contaminants in environmental matrices. However, the determination of trace metals in aqueous samples is difficult due to various factors, particularly their low concentrations and matrix effects. Pre-concentration and separation techniques can solve these problems and lead to a higher confidence level and easy determination of the trace elements. Therefore, several microextraction techniques were developed such as solid phase microextraction (SPME), cloud point extraction (CPE), single drop microextraction (SDME), dispersive liquidliquid microextraction (DLLME) (Pena-Pereira et al., 2009). SDME methodology is based on extracting analytes in a drop of organic solvent that is suspended in the needle tip of a microsyringe, which can be directly immersed in the aqueous sample. After extracting for a set period of time, the organic drop is retracted back into the microsyringe and analyzed by chromatography or spectrometry methods.

DLLME is based on the ternary component solvent systems. It is a simple and fast microextraction technique. For DLLME, an organic solvent with high density such as tetrachlorometane, carbon disulphide, chlorobenzene, and a disperser solvent with high miscibility in both extractant and aqueous phase such as methanol, ethanol, acetonitrile or acetone are used. DLLME, whether conventional or IL-based, is a popular microextraction technique. 


\section{Materials and Methods}

A Perkin-Elmer model AAnalyst 800 atomic absorption spectrophotometer and THGA prolytic coated graphite furnace equipped with a lead hollow-cathode lamp was used for absorbance measurements. Stock solution of $\mathrm{Pb}$ (II) $\quad\left(1000 \mu \mathrm{gmL}^{-1}\right)$ was prepared by dissolving appropriate amount of pure nitrate salt (Merck) in deionized water. The chelating agent Rhodamine B solution obtained from Merck was prepared in deionized water. 1-butyl-3-methylimidazolium hexafluorophosphate $\left(\left[\mathrm{C}_{4} \mathrm{MIM}\right]\left[\mathrm{PF}_{6}\right]\right)$ and diisobuthyl ketonecarbontetrachloride mixture (Merck) were employed as an extractant solvent without further purification. Acetonitrile (Merck) was used as disperser solvent. Phosphoric acid (Sigma), potassium iodide (KI) (Chem $\mathrm{Lab})$, ascorbic acid $\left(\mathrm{C}_{6} \mathrm{H}_{8} \mathrm{O}_{6}\right)$ (Merck) were used for $\mathrm{Pb}$ Rhodamine B complex formation (Shaopu et al., 1983).

\section{Results and Discussion}

The present work describes a novel mixed liquids of diisobutylketone and carbontetrachloride $(1.3: 1 \mathrm{v} / \mathrm{v})$ as an organic acceptor solvent for the preconcentration of lead ions coupling SDME and ETAAS. The method was based on the formation of an ionic pair between lead iodide-Rhodamine $\mathrm{B}$ in phosphoric acid medium. In the presence of a large excess of KI, anionic lead iodide was complexed with Rhodamine B (Shaopu et al., 1983) as an ion-association complex. In the preconcentration step, lead iodide-Rhodamine B complex was extracted into a $10 \mu \mathrm{L}$ diisobutylketone-carbontetrachloride mixture. Some effective parameters on extraction and complex formation, such as $\mathrm{pH}$, type and volume of organic solvent, concentration of complexing agent and extraction time were optimized. Under the optimized conditions, the calibration graphs were linear in the range of 0.025-1.00 $\mu \mathrm{gL}^{-1}$, the detection limit was $0.008 \mu \mathrm{gL}^{-1}$ and the relative standard deviation $(\mathrm{n}=8)$ at $0.25 \mu \mathrm{gL}^{-1} \quad \mathrm{~Pb}$ (II) concentration level was $4.6 \%$. The enhancement factor defined as the slope ratio of two calibration curves with microextraction and without microextraction was 152 .

In the second work, the ionic liquid $\left[\mathrm{C}_{4} \mathrm{MIM}\right]\left[\mathrm{PF}_{6}\right]$, was employed as a solvent for DLLME of lead ions as Rhodamine B complex.

In this method, $1.5 \mathrm{~mL}$ of acetonitrile (disperser solvent) containing $150 \mu \mathrm{L}$ of $\left[\mathrm{C}_{4} \mathrm{MIM}\right]\left[\mathrm{PF}_{6}\right]$, (extraction solvent) was injected rapidly into the sample solution. To attain a high recovery, the effect of different parameters such as $\mathrm{pH}$, type and volume of diluting agent, amounts of extractant and disperser solvents, concentration of chelating agent, concentration of salt, coexisting ions and etc. were optimized. The calibration graph was linear in the range of $0.07-1.00 \mu \mathrm{gL}^{-1}$, the detection limit was $0.0129 \mu \mathrm{gL}^{-1}$ and the relative standard deviation $(\mathrm{n}=8)$ at $0.5 \mu \mathrm{gL}^{-1} \mathrm{~Pb}$ (II) concentration was $2.9 \%$. The obtained enhancement factor for a sample volume of $6 \mathrm{~mL}$ was 89 . The enhancement factor was obtained as the ratio of the slopes of the calibration curves for lead with and without the preconcentration step.

The accuracy of the proposed methods (SDME and DLLME) were verified with the analysis of the Certified Reference Materials. The efficiency of the SDME and ILbased DLLME were validated with the determination of lead in tap water, well water and rice flour.

\section{Conclusion}

In this study, we proposed the use of DLLME and SDME methods for determination of trace levels of lead in different real samples. These preconcentration methods were successfully applied to low concentrations of lead in water and food samples with good accuracy and precision.

\section{References}

Aceto M, Abollino O, Bruzzoniti MC, Mentasti E, Sarzanini C, Melandrino M. Determination of metals in wine with atomic spectroscopy (flameAAS, GF-AAS and ICP-AES); a review. Food Addit Contam 2002; 19: 126.

Ming-Ho Y. Environmental Toxicology: Biological and Health Effects of Pollutants, 2nd edn. CRC Pres 2005, 23.

Pena-Pereira F, Lavilla I, Bendicho C. Miniaturized preconcentration methods based on liquid-liquid extraction and their application in inorganic ultratrace analysis and speciation: A review, Spectrochimica Acta Part B 2009; 64: 15

Shaopu L, Yi L, Zhonfan L. Spectrophotometric

Determination of Trace Amounts of Cadmium and Lead with Iodide and Rhodamine B: Mikrochimica Acta III 983; 355-366.

Xia L, Xuan L, Wu Y, Hu B, Chen R, Ionic liquids based single drop microextraction combined with electrothermal vaporization inductively coupled plasma mass spectrometry for determination of $\mathrm{Co}$, $\mathrm{Hg}$, and $\mathrm{Pb}$ in biological and environmental samples: Spectrochimica Acta Part B 2008; 12901296.

Wang Y, Chen ML, Wang JH, New developments in flow injection/sequential injection on-line separation and preconcentration coupled with electrothermal atomic absorption spectrometry for trace metal analysis, Appl. Spectrosc. Rev. 42 2007; 103-118. 